\title{
Nonequilibrium total dielectric function approach to the electron Boltzmann equation for inelastic scattering in doped polar semiconductors
}

\author{
B.A. Sanborn \\ Semiconductor Electronics Division \\ National Institute of Standards and Technology, Gaithersburg, MD 20899
}

\begin{abstract}
This paper describes a simple and general method for deriving the inelastic collision term in the electron Boltzmann equation for scattering from a coupled electron-phonon system, and applies the method to the case of doped polar semiconductors. In the Born approximation, the inelastic differential scattering rate $W^{\text {inel }}$ can be expressed in terms of the nonequilibrium total dynamic dielectric function, which includes both electronic and lattice contributions. Within the random-phase approximation $W^{\text {inel }}$ separates into two components: an electron-electron interaction containing the nonequilibrium distribution function for excitations of the electron gas, and a Fröhlich interaction including the phonon distribution function and self-energy due to polarization of the electrons. Each of these two interactions is screened by only the electronic part of the total dielectric function which contains the high frequency dielectric constant, unlike commonly used expressions which contain the static dielectric constant. Detailed balance between plasmons and electron-hole pairs in steady state is used to eliminate the nonequilibrium plasmon distribution from the Boltzmann equation, resulting in a dynamically screened electron-electron collision term. The phonon self-energy modifies the longitudinal optical phonon dispersion so that two hybrid normal modes contribute to the electron-phonon collision term.
\end{abstract}

\section{INTRODUCTION}

The electron Boltzmann equation for inelastic scattering in solids is usually established either by making approximations to more general equations derived with powerful nonequilibrium Green's function techniques [1 4 , or simply by ansatz with the aid of analogy to simpler physical systems. An alternative method described here proceeds by using time-dependent perturbation theory to determine the inelastic collision term from the total dynamic dielectric function for the nonequilibrium coupled electron-phonon system. This paper treats the case of a system of polar optical phonons in the presence of conduction electrons and Coulomb interactions, though the method is more generally applicable. Except for the nonequilibrium character of the Boltzmann problem, the approach is similar to Van Hove's correlation function method [5] applied to doped polar semiconductors [6] to determine the inelastic lifetime of a quasiparticle excitation of an equilibrium system. In addition to providing physical insight, the total dielectric function approach is a systematic way to include scattering against coupled electron-phonon modes in the Boltzmann equation, so that the influence of mode coupling on mobility can be studied. Numerical results for mobility in n-type GaAs are presented in reference [7].

In the Born approximation, the interaction between a conduction electron and a coupled electron-phonon system can be represented as an effective electron-electron interaction screened by the total dynamic longitudinal dielectric function, $\epsilon_{T}(\mathbf{q}, \omega)$, which includes contributions from both electrons and phonons [8 10]. In this context, $\epsilon_{T}$ describes the linear response of the electron-phonon gas not to an externally applied potential, but to the internal potential due to the probe electron plus the induced density fluctuations of the gas itself. The effective interaction has the form of a total density autocorrelation function. Unlike the lifetime problem [6], the entire electron-phonon gas is away from equilibrium in the dc transport case, and the correlation function must be evaluated for the nonequilibrium ensemble. It follows that the effective electron-electron interaction is screened by the nonequilibrium total dielectric function in this case. In fact, taking account of the nonequilibrium character of the interaction is essential to obtaining the correct form of the inelastic collision term, which contains nonequilibrium distribution functions for the system's excitations.

The effective electron-electron interaction can be separated into a purely electron-electron term and a screened electron-phonon interaction. In the random-phase approximation (RPA), the electron-phonon part contains a phonon self-energy which arises from the polarization of the electron gas [10]. The self-energy correction modifies the longitudinal optical (LO) phonon dispersion in doped polar semiconductors, producing two hybrid normal modes with phonon strength in both. This paper determines the inelastic differential scattering rate $W^{\text {inel }}$ for the nonequilibrium case in the RPA Born approximation. The result consists of an electron-electron component containing the nonequilibrium distribution function for excitations of the electron gas, and an electron-phonon component containing the phonon self-energy and distribution function. Each of these two interactions is screened by only the electronic part of the total dielectric function, which contains the high-frequency dielectric constant $\epsilon_{\infty}$. Thus, the RPA Born result differs from commonly used expressions for screened interactions in doped polar semiconductors which contain the 
static dielectric constant $\epsilon_{0}$. Examples using $\epsilon_{0}$ rather than $\epsilon_{\infty}$ for n-type GaAs include a calculation of dynamically screened electron-electron scattering [1], as well as static screening approximations for electron-LO phonon scattering [12] and electron-electron scattering [13]. For more strongly polar doped semiconductors (where $\epsilon_{0} \gg \epsilon_{\infty}$ ), it is essential to treat this aspect of the screening problem correctly.

The collective modes have a finite lifetime for decay into electron-hole pairs. This Landau damping process provides an indirect mechanism for single-particle electron-electron scattering. For the electronic system in steady state, Landau damping creates a detailed balance relation between plasmons and electron-hole pairs which can be used to eliminate the nonequilibrium plasmon distribution from the Boltzmann equation in favor of the single-particle electron distribution. In the approximation of no collision damping, the result is an electron-electron collision term screened in the dynamic RPA, containing the effects of electron-plasmon scattering. For direct gap doped semiconductors where Umklapp processes are negligible, this collision term conserves total electron momentum and cannot by itself degrade an electrical current if energy surfaces are spherical. However, by rearranging the electron momentum distribution, electron-plasmon and electron-electron scattering can have a significant indirect effect on the outcomes of other scattering processes.

The low-energy hybrid mode arising from the plasmon-phonon coupling in doped polar semiconductors gives increased low-energy electron-phonon scattering strength compared to the uncoupled case. Numerical calculations including the plasmon-phonon coupling have given shorter inelastic lifetimes [6,14] and enhanced hot electron energy relaxation 15] 16], especially at low temperatures and low doping. A simple way to treat the coupled modes is to use the plasmon-pole approximation [17,18] on the phonon self-energy. This procedure is used here to include scattering against the hybrid modes in the electron-phonon collision term so that mode coupling can be treated with the Boltzmann equation in a computationally efficient manner.

This paper is organized as follows. In section II, $W^{\text {inel }}$ is derived within the Born approximation in terms of the total nonequilibrium dielectric function for the coupled electron-phonon system. The RPA is invoked to separate $W^{\text {inel }}$ into electron-electron and electron-phonon components. Section III deals with the electron Boltzmann equation by eliminating the nonequilibrium plasmon distribution from the electron-electron collision term. The electron-phonon collision term is simplified by applying the plasmon-pole approximation to the phonon self-energy.

\section{INELASTIC DIFFERENTIAL SCATTERING RATE}

\section{A. Electron-phonon gas model}

Consider a probe electron scattering from an interacting gas of electrons and polar optical phonons. Interactions with impurities and acoustic phonons can be added by hand later. The probe electron is treated as a quasiparticle with states $|\mathbf{k}\rangle$, effective mass $m^{*}$, and dispersion relation $E_{k}=\hbar^{2} k^{2} / 2 m^{*}$. The Boltzmann equation relies on the validity of the quasiparticle description by assuming that the energy-momentum relation remains well defined when collisions are included. Interactions with the electron-phonon gas produce transitions between the quasiparticle states, but do not broaden them significantly.

For direct-gap polar semiconductors, local field effects are unimportant so that electrons couple only to the macroscopic electric field set up by the LO phonons, and do not interact with the transverse optical (TO) phonons. The Hamiltonian for the electron-LO phonon gas is 10$]$

$$
\tilde{H}_{g}=\sum_{\mathbf{k}, \sigma} \mathcal{E}_{k} c_{\mathbf{k}, \sigma}^{\dagger} c_{\mathbf{k}, \sigma}+\sum_{\mathbf{q}} \hbar \omega_{T O}\left(a_{\mathbf{q}}^{\dagger} a_{\mathbf{q}}+\frac{1}{2}\right)+\frac{1}{2} \sum_{\mathbf{q} \neq 0} v_{q}^{\infty}\left[\rho_{T}^{\dagger}(\mathbf{q}) \rho_{T}(\mathbf{q})-N\right]
$$

where

$$
v_{q}^{\infty}=\frac{v_{q}}{\epsilon_{\infty}}=\frac{4 \pi e^{2}}{q^{2} \epsilon_{\infty}}
$$

and where the electron and LO phonon annihilation operators are denoted by $c_{\mathbf{k}}$ and $a_{\mathbf{q}}$, respectively, $\sigma$ labels the electron spin, and $\omega_{T O}$ is the TO phonon frequency. The relevant phonon frequency in $\tilde{H}_{g}$ is the bare one, $\omega_{T O}$. The ion-ion Coulomb interactions explicitly included in $\tilde{H}_{g}$ shift $\omega_{T O}$ to the longitudinal optical frequency $\omega_{L O}$, so that only $\omega_{L O}$ appears in expressions for observables [10]. The first and second sums in $\tilde{H}_{g}$ describe the noninteracting systems of electrons and phonons, respectively. The last sum includes electron-electron, electron-phonon, and ion-ion Coulomb interactions. The total density operator $\rho_{T}$ is the summation of the density operators of the electrons and LO phonons, 


$$
\rho_{T}(\mathbf{q})=\Omega^{-1 / 2} \rho(\mathbf{q})+Z q\left(\frac{n \hbar}{2 M \omega_{T O}}\right)^{1 / 2} A(\mathbf{q})
$$

where $A(\mathbf{q})=a_{\mathbf{q}}+a_{-\mathbf{q}}^{\dagger}, M$ is the reduced mass of the basis ions, $Z e$ is the effective ionic charge, $n$ is the electron density, $\Omega$ is the system volume, and the Fourier components of the electron density operator are

$$
\rho(\mathbf{q})=\sum_{i} e^{-i \mathbf{q} \cdot \mathbf{r}_{i}}=\sum_{\mathbf{k}, \sigma} c_{\mathbf{k}+\mathbf{q}, \sigma}^{\dagger} c_{\mathbf{k}, \sigma}
$$

The electron self-interaction has been removed from $\tilde{H}_{g}$ by subtracting the term with $N$, the number of electrons in the system. The influence of interband electronic transitions is taken into account by screening the Coulomb interaction $v_{q}$ with the high-frequency dielectric constant $\epsilon_{\infty}$.

The effects of screening by conduction electrons and LO phonons are contained in the interaction term in $\tilde{H}_{g}$ and will appear explicitly in the total dielectric function below. The RPA or mean-field approximation for the interaction term is made by working with eigenstates $|n\rangle$ of the Hamiltonian $H_{g}$ which neglects the terms in $\tilde{H}_{g}$ that are quadratic in density fluctuations $\rho_{T}-\left\langle\rho_{T}\right\rangle[9]$.

$$
\begin{aligned}
H_{g}|n\rangle & =E_{n}|n\rangle \\
H_{g} & =\tilde{H}_{g}-\frac{1}{2} \sum_{\mathbf{q}} v_{q}^{\infty}\left[\rho_{T}^{\dagger}(\mathbf{q})-\left\langle\rho_{T}^{\dagger}(\mathbf{q})\right\rangle\right]\left[\rho_{T}(\mathbf{q})-\left\langle\rho_{T}(\mathbf{q})\right\rangle\right] .
\end{aligned}
$$

$H_{g}$ includes Coulomb interactions at the Hartree level. Excitations of the electron-phonon gas described by $H_{g}$ include both electron-hole pair and collective excitations.

Define $P(n, \mathbf{k} \rightarrow m, \mathbf{k}-\mathbf{q})$ as the probability per unit time for the probe electron to make a transition from $|\mathbf{k}\rangle$ to $|\mathbf{k}-\mathbf{q}\rangle$ while the electron-phonon gas makes a transition from $|n\rangle$ to $|m\rangle$, assuming that initially $|\mathbf{k}\rangle$ is occupied and $|\mathbf{k}-\mathbf{q}\rangle$ is unoccupied. The interactions included in $H_{g}$ produce charge density correlations, resulting in an interaction potential between gas excitations and probe electron which is screened and assumed to be weak. Using Fermi's Golden rule (or the Born approximation) with the probe particle plane wave states $|\mathbf{k}\rangle$, the transition probability is

$$
\begin{aligned}
P(n, \mathbf{k} \rightarrow m, \mathbf{k}-\mathbf{q}) & =\frac{2 \pi}{\hbar}\left(\frac{v_{q}^{\infty}}{\Omega}\right)^{2}\left|\left\langle m\left|\rho_{T}^{\dagger}(\mathbf{q})\right| n\right\rangle\right|^{2} \delta\left(E_{n}-E_{m}+\hbar \omega_{k, k-q}\right) \\
& =\left(\frac{v_{q}^{\infty}}{\Omega \hbar}\right)^{2} \int_{-\infty}^{\infty} d t e^{i \omega_{k, k-q} t}\left\langle n\left|\rho_{T}(\mathbf{q}, t)\right| m\right\rangle\left\langle m\left|\rho_{T}^{\dagger}(\mathbf{q}, 0)\right| n\right\rangle \\
\rho_{T}(\mathbf{q}, t) & =e^{i H_{g} t / \hbar} \rho_{T}(\mathbf{q}) e^{-i H_{g} t / \hbar},
\end{aligned}
$$

where $\omega_{k, k-q}=\hbar\left[k^{2}-(\mathbf{k}-\mathbf{q})^{2}\right] / 2 m^{*}$.

The inelastic differential scattering rate $W^{\text {inel }}(\mathbf{k}, \mathbf{k}-\mathbf{q})$ is the probability per unit time for an electronic transition from the occupied state $|\mathbf{k}\rangle$ to the unoccupied state $|\mathbf{k}-\mathbf{q}\rangle$ due to scattering from the electron-phonon gas. It is found by summing $P(n, \mathbf{k} \rightarrow m, \mathbf{k}-\mathbf{q})$ over all possible final states $|m\rangle$ of the gas and averaging over its initial states $|n\rangle$. For the mobility problem, the average must be taken over the nonequilibrium gas ensemble.

\section{B. Nonequilibrium ensemble averages}

Consider that an electric potential $U(\mathbf{r}, t)$ is applied to the system of probe electron plus electron-phonon gas. This influence is included by adding the term

$$
H_{e x t}(t)=e \int d^{3} r U(\mathbf{r}, t) \rho_{T}(\mathbf{r}, t)
$$

to the Hamiltonian of the system. $H_{e x t}$ is independent of time in steady state, but it is useful to require the potential to be switched on at a time in the distant past when the gas was in thermal equilibrium. This requirement makes it possible to use the Kadanoff and Baym method [19] for evaluating a nonequilibrium expectation value. This method uses the equilibrium density matrix to evaluate an ensemble average at a time after $U$ has been switched on by using the interaction picture to include the dependence on $H_{\text {ext }}$ explicitly. The nonequilibrium expectation value of an operator $\mathcal{O}(\mathbf{r}, t)$ is 19 


$$
\begin{aligned}
\langle\mathcal{O}(\mathbf{r}, t)\rangle_{\text {noneq }} & =\left\langle\mathcal{O}_{U}(\mathbf{r}, t)\right\rangle=\mathcal{Z}^{-1} \operatorname{Tr}\left\{e^{-\beta H_{g}} \mathcal{O}_{U}(\mathbf{r}, t)\right\} \\
\mathcal{O}_{U}(\mathbf{r}, t) & =\mathcal{V}^{-1}(t) \mathcal{O}(\mathbf{r}, t) \mathcal{V}(t), \\
\mathcal{V}(t) & =\mathcal{T} \exp \left[\frac{-i}{\hbar} \int_{-\infty}^{t} d t^{\prime} H_{\text {ext }}\left(t^{\prime}\right)\right]
\end{aligned}
$$

where \langle\rangle denotes an average over the equilibrium population of the electron-phonon gas, $\beta=1 / k_{B} T, \mathcal{Z}=\operatorname{Tr}\left\{e^{-\beta H_{g}}\right\}$ is the partition function for the equilibrium gas, and $\mathcal{T}$ denotes the time ordering operator which orders operators with earliest times to the right.

In the presence of $U(\mathbf{r}, t)$, the transition rate $P(n, \mathbf{k} \rightarrow m, \mathbf{k}-\mathbf{q})$ in the Born approximation is unchanged from its equilibrium form (7) except that the influence of $H_{\text {ext }}$ must be included in the time dependence of the matrix element. Using equations (11. 13) to determine $W^{\text {inel }}(\mathbf{k}, \mathbf{k}-\mathbf{q})$ from $P(n, \mathbf{k} \rightarrow m, \mathbf{k}-\mathbf{q})$ for the nonequilibrium case yields

$$
\begin{aligned}
W^{i n e l}(\mathbf{k}, \mathbf{k}-\mathbf{q}) & =\left(\frac{v_{q}^{\infty}}{\Omega \hbar}\right)^{2} N S_{T U}\left(\mathbf{q}, \omega_{k, k-q}\right), \\
S_{T U}(\mathbf{q}, \omega) & =\frac{1}{N} \int_{-\infty}^{\infty} d t e^{i \omega t}\left\langle\rho_{T U}(\mathbf{q}, t) \rho_{T U}(-\mathbf{q}, 0)\right\rangle \\
\rho_{T U}(\mathbf{q}, t) & =\mathcal{V}^{-1}(t) e^{i H_{g} t / \hbar} \rho_{T} e^{-i H_{g} t / \hbar} \mathcal{V}(t),
\end{aligned}
$$

where $S_{T U}(\mathbf{q}, \omega)$ is the nonequilibrium total dynamic structure factor or spectral function for $\rho_{T U}$, describing the density fluctuation excitations of the nonequilibrium electron-phonon gas. Similarly, the time-reversed rate is

$$
W^{\text {inel }}(\mathbf{k}-\mathbf{q}, \mathbf{k})=\left(\frac{v_{q}^{\infty}}{\Omega \hbar}\right)^{2} N S_{T U}\left(-\mathbf{q},-\omega_{k, k-q}\right) .
$$

When the gas excitations are not too highly damped so that they may usefully be regarded as elementary excitations, and when the deviations from equilibrium are slowly varying in space and time on a scale determined by the frequencies and decay rates of the elementary excitations, it is possible to define nonequilibrium distribution functions and response functions [1,3]. The imaginary (dissipative) part of the response function for the nonequilibrium coupled electronphonon system is

$$
\begin{aligned}
\operatorname{Im}\left[\chi_{T U}(\mathbf{q}, \omega)\right] & =\frac{-1}{2 \hbar \Omega} \int_{-\infty}^{\infty} d t e^{i \omega t}\left\langle\left[\rho_{T U}(\mathbf{q}, t), \rho_{T U}(-\mathbf{q}, 0)\right]\right\rangle \\
& =\frac{-n}{2 \hbar}\left[S_{T U}(\mathbf{q}, \omega)-S_{T U}(-\mathbf{q},-\omega)\right]
\end{aligned}
$$

where $\left[\rho, \rho^{\prime}\right]$ is the commutator $\rho \rho^{\prime}-\rho^{\prime} \rho$.

In general, there is no simple relation between a nonequilibrium spectral function and corresponding response function like the fluctuation-dissipation theorem (FDT) for the equilibrium case:

$$
S_{T}(\mathbf{q}, \omega)=\frac{-2 \hbar}{n}\left[N^{0}(\omega)+1\right] \operatorname{Im}\left[\chi_{T}(\mathbf{q}, \omega)\right],
$$

where $N^{0}(\omega)=\left(e^{\beta \hbar \omega}-1\right)^{-1}$ is the equilibrium Bose-Einstein distribution function, while $S_{T}$ and $\operatorname{Im} \chi_{T}$ are the equilibrium forms of (15) and (18). In particular, since the relation between the time-reversed nonequilibrium spectral functions $S_{T U}(\mathbf{q}, \omega)$ and $S_{T U}(-\mathbf{q},-\omega)$ is not known, $\operatorname{Im}\left[\chi_{T U}(\mathbf{q}, \omega)\right]$ is not determined by $S_{T U}(\mathbf{q}, \omega)$ alone. It is possible, of course, to define unknown dimensionless functions $N^{>}(\mathbf{q}, \omega)$ and $N^{<}(\mathbf{q}, \omega)$ such that

$$
\begin{aligned}
S_{T U}(\mathbf{q}, \omega) & =\frac{-2 \hbar}{n} N^{>}(\mathbf{q}, \omega) \operatorname{Im}\left[\chi_{T U}(\mathbf{q}, \omega)\right] \\
S_{T U}(-\mathbf{q},-\omega) & =\frac{-2 \hbar}{n} N^{<}(\mathbf{q}, \omega) \operatorname{Im}\left[\chi_{T U}(\mathbf{q}, \omega)\right] .
\end{aligned}
$$

Equations (19)and (21)-(22) then imply

$$
N^{>}(\mathbf{q}, \omega)-N^{<}(\mathbf{q}, \omega)=1 .
$$

Adopting the notation $N^{<}(\mathbf{q}, \omega)=N(\mathbf{q}, \omega)$, we have from (14), 21), and (23), 


$$
W^{\text {inel }}(\mathbf{k}, \mathbf{k}-\mathbf{q})=\frac{-2\left(v_{q}^{\infty}\right)^{2}}{\Omega \hbar}\left[N\left(\mathbf{q}, \omega_{k, k-q}\right)+1\right] \operatorname{Im}\left[\chi_{T U}\left(\mathbf{q}, \omega_{k, k-q}\right)\right] .
$$

Equivalently, the Born aproximation for $W^{\text {inel }}$ can be expressed in terms of the imaginary part of the total screened Coulomb interaction $v_{q} / \epsilon_{T U}$ between the probe particle and the electron-phonon gas,

$$
W^{\text {inel }}(\mathbf{k}, \mathbf{k}-\mathbf{q})=\frac{-2 v_{q}}{\Omega \hbar}\left[N\left(\mathbf{q}, \omega_{k, k-q}\right)+1\right] \operatorname{Im}\left[\epsilon_{T U}^{-1}\left(\mathbf{q}, \omega_{k, k-q}\right)\right] .
$$

This follows from the relations between the total susceptibility $\chi_{T U}(\mathbf{q}, \omega)$, polarization $P_{T U}(\mathbf{q}, \omega)$, and dielectric function $\epsilon_{T U}(\mathbf{q}, \omega)$ for the nonequilibrium coupled system,

$$
\begin{array}{r}
\chi_{T U}(\mathbf{q}, \omega)=\frac{P_{T U}(\mathbf{q}, \omega)}{1-v_{q}^{\infty} P_{T U}(\mathbf{q}, \omega)} \\
\epsilon_{T U}(\mathbf{q}, \omega)=\epsilon_{\infty}\left[1-v_{q}^{\infty} P_{T U}(\mathbf{q}, \omega)\right] .
\end{array}
$$

The relation $\left(v_{q}^{\infty}\right)^{2} \operatorname{Im} \chi_{T U}(\mathbf{q}, \omega)=v_{q} \operatorname{Im} \epsilon_{T U}^{-1}$ follows from (26) and (27), and yields (25) from (24).

The weight factor $N(\mathbf{q}, \omega)$ in (25) plays the role of the distribution function for the nonequilibrium excitations of the coupled electron-phonon system. Just as for the equilibrium case in equation (20), the structure factor has been written as a product of a function $\left(\operatorname{Im}\left[\epsilon_{T}^{-1}\right]\right)$ describing the strength of interactions with the electron phonon gas, and a function giving the occupation probabilities for the gas excitations. However, it should be emphasized that the FDT has not been used to derive (25) and $N(\mathbf{q}, \omega)$ is an unknown function.

\section{Separation of the total interaction}

The total nonequilibrium structure factor $S_{T U}$ defined by (15) is exactly separable into a purely electronic part $S_{U}^{e}$ plus the remainder $S_{U}^{p h}$ which includes electron-phonon and ion-ion Coulomb interactions. Using the nonequilibrium version of the total density operator (3),

$$
\begin{aligned}
S_{T U}(\mathbf{q}, \omega) & =S_{U}^{e}(\mathbf{q}, \omega)+S_{U}^{p h}(\mathbf{q}, \omega) \\
S_{U}^{e}(\mathbf{q}, \omega) & =\frac{1}{\Omega N} \int_{-\infty}^{\infty} d t e^{i \omega t}\left\langle\rho_{U}(\mathbf{q}, t) \rho_{U}(-\mathbf{q}, 0)\right\rangle \\
S_{U}^{p h}(\mathbf{q}, \omega) & =\frac{1}{N} \int_{-\infty}^{\infty} d t e^{i \omega t}\left\{Z q ( \frac { n \hbar } { 2 M \omega _ { T O } \Omega } ) ^ { 1 / 2 } \left[\left\langle\rho_{U}(\mathbf{q}, t) A_{U}(-\mathbf{q}, 0)\right\rangle\right.\right. \\
& \left.\left.+\left\langle A_{U}(\mathbf{q}, t) \rho_{U}(-\mathbf{q}, 0)\right\rangle\right]+(Z q)^{2} \frac{n \hbar}{2 M \omega_{T O}}\left\langle A_{U}(\mathbf{q}, t) A_{U}(-\mathbf{q}, 0)\right\rangle\right\} .
\end{aligned}
$$

This section relates $S^{e}$ and $S^{p h}$ to corresponding electron and phonon parts of the response function, just as (21) relates the total structure factor to the total response function. To do so, equation (25) must be split into electronelectron and electron-phonon parts. Imposing the RPA for the dielectric function is a crucial step towards this goal. The response of the coupled system in the RPA is the sum of the electron and ion response taken separately [9, 20]. In this approximation, the total screened interaction $v_{q} / \epsilon_{T U}$ is the sum of a purely electron-electron part and an electron-phonon interaction that includes a phonon self-energy due to the polarization of the conduction electrons 21.

$$
\frac{v_{q}}{\epsilon_{T U}}=\frac{v_{q}^{\infty}}{\epsilon_{U}}+\frac{M_{q}^{2}}{\left|\epsilon_{U}\right|^{2}} D_{U}(\mathbf{q}, \omega) .
$$

The electron-electron part of the total interaction is $v_{q}^{\infty} / \epsilon_{U}$, where $\epsilon_{U}$ is the nonequilibrium RPA dielectric function determined by electron-electron interactions only. The polarization for the noninteracting electron gas $P^{(1)}$ has the same Lindhard form as the equilibrium case, except that the nonequilibrium electron distribution function $f$ is used instead of the Fermi-Dirac function [3].

$$
\begin{aligned}
\epsilon_{U}(\mathbf{q}, \omega) & =1-v_{q}^{\infty} P^{(1)}(\mathbf{q}, \omega) \\
P^{(1)}(\mathbf{q}, \omega) & =\frac{1}{\Omega} \sum_{p, \sigma} \frac{f(\mathbf{p})-f(\mathbf{p}+\mathbf{q})}{\hbar \omega_{p, p+q}-\hbar \omega-i \delta} .
\end{aligned}
$$


The remaining part of the total interaction is a product of the screened electron-phonon matrix element $M_{q}^{2}\left|\epsilon_{U}\right|^{-2}$, and the nonequilibrium phonon Green's function $D_{U}(\mathbf{q}, \omega)$ containing the self-energy correction $M_{q}^{2} D_{U}^{(0)} \chi_{U}$ to the noninteracting Green's function $D_{U}^{(0)}$.

$$
\begin{aligned}
M_{q}^{2} & =v_{q} \frac{\hbar \omega_{L O}^{2}}{2 \omega_{T O}}\left(\frac{1}{\epsilon_{\infty}}-\frac{1}{\epsilon_{0}}\right) \\
D_{U}(\mathbf{q}, \omega) & =\frac{D_{U}^{(0)}}{1-M_{q}^{2} D_{U}^{(0)} \chi_{U}(\mathbf{q}, \omega)} .
\end{aligned}
$$

Now define unknown dimensionless functions $N_{e}^{\gtrless}$ and $N_{p h}^{<}$to relate the electron and phonon components of $S_{T U}(\mathbf{q}, \omega)$ in $(28)$ to the respective components of the imaginary part of $v_{q} \epsilon_{T U}^{-1}$ in (32).

$$
\begin{array}{r}
\left(v_{q}^{\infty}\right)^{2} S_{U}^{e}( \pm \mathbf{q}, \pm \omega)=\frac{-2 \hbar}{n} v_{q}^{\infty} N_{e}^{\gtrless}(\mathbf{q}, \omega) \operatorname{Im}\left[\epsilon_{U}^{-1}(\mathbf{q}, \omega)\right] \\
\left(v_{q}^{\infty}\right)^{2} S_{U}^{p h}( \pm \mathbf{q}, \pm \omega)=\frac{-2 \hbar}{n} \frac{M_{q}^{2}}{\left|\epsilon_{U}\right|^{2}} N_{p h}^{>}(\mathbf{q}, \omega) \operatorname{Im}\left[D_{U}(\mathbf{q}, \omega)\right] .
\end{array}
$$

Finally, using (14), 28), (37), and (38),

$$
\begin{aligned}
W^{i n e l}(\mathbf{k}, \mathbf{k}-\mathbf{q})=\frac{-2}{\Omega \hbar}\left\{v_{q}^{\infty}\left[N_{e}\left(\mathbf{q}, \omega_{k, k-q}\right)+1\right] \operatorname{Im}\left[\epsilon_{U}^{-1}\left(\mathbf{q}, \omega_{k, k-q}\right)\right]\right. \\
\left.\quad+\frac{M_{q}^{2}}{\left|\epsilon_{U}\left(q, \omega_{k, k-q}\right)\right|^{2}}\left[N_{p h}\left(\mathbf{q}, \omega_{k, k-q}\right)+1\right] \operatorname{Im}\left[D_{U}\left(\mathbf{q}, \omega_{k, k-q}\right)\right]\right\} .
\end{aligned}
$$

Within the RPA, the expressions (25) and (39) for $W^{\text {inel }}$ are equivalent. The last expression contains two unknown functions, $N_{e}$ and $N_{p h}$, playing the roles of the nonequilibrium occupation functions for the excitations of the electronic and lattice components of the coupled system. Notice that $N_{e}$ must describe a distribution with boson properties. This is because the density fluctuation $\rho_{q}^{\dagger}$ produces excitations of the electron gas which conserve the total number of electrons, such as electron-hole pairs and plasmons, which have boson properties.

\section{ELECTRON BOLTZMANN EQUATION}

The time rate of change of the nonequilibrium electron distribution $f(\mathbf{k})$ due to collisions is determined by the differential scattering rate $W(\mathbf{k}, \mathbf{k}-\mathbf{q})$; i.e., the probability per unit time for the transition $|\mathbf{k}\rangle \rightarrow|\mathbf{k}-\mathbf{q}\rangle$. The depletion rate of $f(\mathbf{k})$ is just $W(\mathbf{k}, \mathbf{k}-\mathbf{q})$ weighted by the probabilities that $|\mathbf{k}\rangle$ is occupied and $|\mathbf{k}-\mathbf{q}\rangle$ is unoccupied, summed over all possible final states. The collision term, $-\{\dot{f}(\mathbf{k})\}_{\text {coll }}$, in the Boltzmann equation is this depletion rate minus the replenishment rate determined similarly. The electric field in the sample is $\mathbf{F}(\mathbf{r}, \mathbf{t})=-\nabla[U(\mathbf{r}, t)+$ $\left.\left\langle U_{\text {ind }}(\mathbf{r}, t)\right\rangle\right]$, where $\left\langle U_{\text {ind }}\right\rangle$ is the average induced potential resulting from the system's response to the applied potential $U$. Thus, for a homogeneous system under the influence of a static electric field $\mathbf{F}$,

$$
\begin{aligned}
& \frac{-e \mathbf{F}}{\hbar} \cdot \frac{\partial f(\mathbf{k})}{\partial \mathbf{k}}=-\{\dot{f}(\mathbf{k})\}_{\text {coll }} \\
= & \frac{1}{\Omega} \sum_{\mathbf{q}}\{W(\mathbf{k}, \mathbf{k}-\mathbf{q}) f(\mathbf{k})[1-f(\mathbf{k}-\mathbf{q})]-W(\mathbf{k}-\mathbf{q}, \mathbf{k}) f(\mathbf{k}-\mathbf{q})[1-f(\mathbf{k})]\} .
\end{aligned}
$$

$W(\mathbf{k}, \mathbf{k}-\mathbf{q})$ is a sum of rates for elastic and inelastic processes, $W=W^{e l}+W^{i n e l}$. Using the separation of the inelastic rate $W_{\text {inel }}$ in (39), the inelastic contribution to the collision term separates into electron-electron and electron-phonon collision terms,

$$
\{\dot{f}\}_{\text {coll }}^{\text {inel }}=\{\dot{f}\}_{\text {coll }}^{e e}+\{\dot{f}\}_{\text {coll }}^{e p} .
$$




\section{A. Electron-electron collision term}

The purely electronic component of $W^{\text {inel }}$ in (39) gives the collision term

$$
\begin{aligned}
& -\{\dot{f}(\mathbf{k})\}_{\text {coll }}^{e e}= \\
& -\frac{2}{\hbar \Omega^{2}} \sum_{\mathbf{q}} v_{q}^{\infty}\left\{\left[N_{e}\left(\mathbf{q}, \omega_{k, k-q}\right)+1\right] \operatorname{Im}\left[\epsilon_{U}^{-1}\left(\mathbf{q}, \omega_{k, k-q}\right)\right] f(\mathbf{k})[1-f(\mathbf{k}-\mathbf{q})]\right. \\
& \left.\quad-N_{e}\left(\mathbf{q}, \omega_{k, k-q}\right) \operatorname{Im}\left[\epsilon_{U}^{-1}\left(\mathbf{q}, \omega_{k, k-q}\right)\right] f(\mathbf{k}-\mathbf{q})[1-f(\mathbf{k})]\right\}
\end{aligned}
$$

The next step towards putting the collision term into a useful form is to eliminate the unknown function $N_{e}$, which gives the occupation probabilities of the density fluctuation excitations of the electron system. This step is straightforward if an association is made between $N_{e}$ and the nonequilibrum plasmon distribution, denoted here by $N_{p}$. In steady state, there exists a local detailed balance (LDB) between plasmons and electron-hole pairs which can be used to eliminate $N_{e}$ in favor of $f$ in the electron-electron collision term [3,22]. The LDB condition is equivalent to the FDT for the nonequilibrium ensemble [23] which applies when $\chi$ determines the response not to external forces, but to internal fluctuation forces. It holds when the Landau damping rate $\gamma=2 \operatorname{Im} P^{(1)}$ is large compared to the time variation of $f$, so that the plasmons come into equilibrium with the electrons before $f$ changes appreciably. For the mobility problem, $f$ is stationary and the condition always holds exactly, except at small $q$ values (where $\gamma$ vanishes) which do not contribute to the electron collision integral because of energy and momentum conservation restrictions. If LDB is used in combination with the "collisionless damping" approximation [3], equation (42) is equivalent to the standard single-particle electron-electron collision term, but screened with the dynamic RPA dielectric function. This may be shown easily for the case of weak damping, in which the plasmons are well-defined excitations, by considering the Boltzmann equation for the nonequilibrium plasmon distribution $N_{p}(q)=N_{p}\left(\mathbf{q}, \omega_{q}\right)$ with $\omega_{q}=\omega_{p}(q)$ [22,24. Plasmons are represented by the peaks in the spectral function $\operatorname{Im} \epsilon_{U}^{-1}$. In the RPA, this function contains no corrections for collision broadening, so that the relaxation of $N_{p}(q)$ is completely described by coherent absorption and emission of electron-hole pairs.

$$
\begin{aligned}
\dot{N}_{p}(q)=\frac{\pi v_{q} \omega_{q}}{\Omega^{2}} \sum_{\mathbf{k}}\{ & {\left[N_{p}(q)+1\right] f(\mathbf{k}+\mathbf{q})[1-f(\mathbf{k})] } \\
& \left.-N_{p}(q) f(\mathbf{k})[1-f(\mathbf{k}+\mathbf{q})]\right\} \delta\left(\hbar \omega_{k, k+q}-\hbar \omega_{q}\right) .
\end{aligned}
$$

In steady state, $\dot{N}_{p}=0$. Therefore, the relation between the distributions $N_{p}$ and $f$ is necessarily

$$
\begin{array}{r}
N_{p}(q) \operatorname{Im}\left[P_{U}^{(1)}\left(\mathbf{q}, \omega_{q}\right)\right]=\frac{n}{2 \hbar} S_{U}^{(1)}\left(\mathbf{q}, \omega_{q}\right) \\
\operatorname{Im}\left[P_{U}^{(1)}(\mathbf{q}, \omega)\right]=\frac{2 \pi}{\Omega} \sum_{\mathbf{p}}[f(\mathbf{p})-f(\mathbf{p}+\mathbf{q})] \delta\left(\hbar \omega_{p, p+q}-\hbar \omega\right) \\
S_{U}^{(1)}(\mathbf{q}, \omega)=\frac{4 \pi \hbar}{N} \sum_{\mathbf{p}} f(\mathbf{p}+\mathbf{q})[1-f(\mathbf{p})] \delta\left(\hbar \omega_{p, p+q}-\hbar \omega\right),
\end{array}
$$

relating the response function $\operatorname{Im}\left[P_{U}^{(1)}\right]$ and structure factor $S_{U}^{(1)}$ for the noninteracting nonequilibrium electron gas.

The detailed balance relation between plasmons and electron-hole pairss simplifies the electron-electron collision term (42), if we assume $N_{e}=N_{p}$. Using the RPA interaction $\operatorname{Im}\left[\epsilon_{U}^{-1}\right]=v_{q}^{\infty}\left|\epsilon_{U}\right|^{-2} \operatorname{Im}\left[P_{U}^{(1)}\right]$ and (44 46) gives

$$
\begin{aligned}
-\left\{\dot{f}\left(\mathbf{k}_{1}\right)\right\}_{\text {coll }}^{e e}=\frac{4 \pi}{\hbar \Omega^{3}} \sum_{2,3,4} \frac{\left(v_{q}^{\infty}\right)^{2}}{\left|\epsilon_{U}\left(q, \frac{E_{1}-E_{3}}{\hbar}\right)\right|^{2}} & \delta\left(E_{1}+E_{2}-E_{3}-E_{4}\right) \delta\left(\mathbf{k}_{1}+\mathbf{k}_{2}-\mathbf{k}_{3}-\mathbf{k}_{4}\right) \\
\times & \left\{f\left(\mathbf{k}_{1}\right) f\left(\mathbf{k}_{2}\right)\left[1-f\left(\mathbf{k}_{3}\right)\right]\left[1-f\left(\mathbf{k}_{4}\right)\right]\right. \\
& \left.-\left[1-f\left(\mathbf{k}_{1}\right)\right]\left[1-f\left(\mathbf{k}_{2}\right)\right] f\left(\mathbf{k}_{3}\right) f\left(\mathbf{k}_{4}\right)\right\},
\end{aligned}
$$


when the notational changes $\mathbf{k} \rightarrow \mathbf{k}_{1}, \mathbf{p} \rightarrow \mathbf{k}_{2}$ are made, the summation variable $\mathbf{q}$ is changed to $\mathbf{k}_{3}=\mathbf{k}_{1}-\mathbf{q}$, and a summation over a delta function for momentum conservation is included. Notice that the nonequilibrium inverse dielectric function was needed to obtain the collision term with nonequilibrium distributions for all four (incoming and outgoing) electrons. The remaining factor of $\left|\epsilon_{U}\right|^{2}$ in equation (47) appears only in its equilibrium form $|\epsilon|^{2}$ if the Boltzmann equation is linearized with respect to the field strength $\mathbf{F}$, since the other factors vanish in equilibrium. Also notice that $\epsilon_{\infty}$ rather than $\epsilon_{0}$ appears in the electronic dielectric function $\epsilon_{U}(q, \omega)$ (see equation (33)) which screens the interaction in the electron-electron collision term (47), unlike some previous calculations using $\epsilon_{0}$ for electron-electron scattering in GaAs 11, 13.

It should be emphasized that (47) includes the effect of electron-plasmon scattering through the resonance in $\epsilon(q, \omega)$ at $\omega=\omega_{q}$. For dc transport, the two-step process:

$$
\text { electron - hole pair } \rightarrow \text { plasmon } \rightarrow \text { electron }- \text { hole pair }
$$

is equivalent to a dynamically screened electron-electron scattering event because of the detailed balance between plasmons and electrons. Since (47) conserves total electron momentum (in the absence of Umklapp processes), its effect on the electrical current in materials with spherical energy surfaces is nonvanishing only because it rearranges the momentum distribution, which influences other scattering processes. The most important difference between using (47) and a previous treatment [25] of the effects of electron-plasmon scattering on mobility is that the earlier work assumed an equilibrium (Bose-Einstein) distribution function for the plasmons. In the latter case, there is a net momentum loss from the nonequilibrium electron system.

\section{B. Electron-phonon collision term}

If the phonons interacted only with electrons by non-Umklapp processes, they would reach a local detailed balance with the electrons just as the plasmons do. But, in fact, the phonons interact with other phonons through anharmonic lattice forces. The excess momentum given to the LO phonons by the nonequilibrium electron system is dissipated primarily through interactions with acoustic phonons, which can lose momentum to the environment through umklapp processes. Generally, mobility calculations have employed the "Bloch assumption" that the phonon system may be treated as if it were in thermal equilibrium [26], valid when anharmonic processes are much faster than electron-phonon scattering.

Adopting the model of equilibrium phonons, the electron-phonon component of $W^{\text {inel }}$ in (39) gives the collision term,

$$
\begin{aligned}
& -\{\dot{f}(\mathbf{k})\}_{\text {coll }}^{e p}= \\
& -\frac{2}{\hbar \Omega^{2}} \sum_{\mathbf{q}} \frac{M_{q}^{2}}{\left|\epsilon_{U}\left(q, \omega_{k, k-q}\right)\right|^{2}}\left\{\left[N^{0}\left(\omega_{k, k-q}\right)+1\right] \operatorname{Im} D\left(q, \omega_{k, k-q}\right) f(\mathbf{k})[1-f(\mathbf{k}-\mathbf{q})]\right. \\
& \left.-N^{0}\left(\omega_{k, k-q}\right) \operatorname{Im} D\left(q, \omega_{k, k-q}\right) f(\mathbf{k}-\mathbf{q})[1-f(\mathbf{k})]\right\} .
\end{aligned}
$$

The electron-phonon matrix element is screened by only the electronic part of the total dynamic dielectric function. The long-wavelength, static limit of the RPA $\epsilon(q, \omega)$ is the temperature-dependent Thomas-Fermi dielectric function with the high-frequency dielectric constant $\epsilon_{\infty}$. Therefore, equation (48) in this limit gives a somewhat different result than treatments of electron-LO phonon scattering using Thomas-Fermi screening with the static dielectric constant $\epsilon_{0}$ [12]. For the nondegenerate case, the long wavelength limit of (48) is in agreement with the original treatment of screening in polar semiconductors by Ehrenreich [27.

The ion-ion Coulomb interactions included in $\tilde{H}_{g}$ (equation (11) make a self-energy contribution to the phonon Green's function [10]. The real part of this contribution shifts the LO phonon energy from $\hbar \omega_{T O}$ to $\hbar \omega_{L O}$ in the denominator of the noninteracting Green's function $D^{(0)}=-2 \omega_{T O} / \hbar\left(\omega_{L O}^{2}-\omega^{2}\right)$. Damping due to anharmonic interactions and disorder is neglected here.

The interacting phonon Green's function

$$
D(q, \omega)=\frac{D^{(0)}}{1-M_{q}^{2} D^{(0)} \chi(q, \omega)}=\frac{2 \omega_{T O} / \hbar}{\omega^{2}-\omega_{L O}^{2}-2 \omega_{T O} M_{q}^{2} \chi(q, \omega) / \hbar}
$$

contains the self-energy term $\Pi^{e p}=M_{q}^{2} D^{(0)} \chi$ due to the polarization of the electron gas. The real part of $\Pi^{e p}$ renormalizes the LO phonon frequency, giving rise to two hybrid modes of mixed electron-phonon character. The 
coupled modes have been treated previously [18] with the finite-temperature plasmon-pole approximation for $\chi$ [17]. In this approximation, the electronic excitation spectrum which couples to the phonons is represented by a single mode with energy varying between $\hbar \omega_{p}=\sqrt{4 \pi n e^{2} / m^{*} \epsilon_{\infty}}$ at small $q$ and $h^{2} q^{2} / 2 m^{*}$ at large $q$. The plasmon coupling strength and energy are determined by requiring fulfillment of the f-sum rule and zero-frequency Kramers-Kronig relation, so that the plasmon-pole approximation for $\chi$ is [17, 18]

$$
\begin{aligned}
\chi^{p p}(q, \omega) & =\frac{1}{v_{q}^{\infty}} \frac{\omega_{p}^{2}}{\omega^{2}-\tilde{\omega}_{p}^{2}} \\
\tilde{\omega}_{p}^{2} & =\omega_{p}^{2}\left[1-\epsilon^{-1}(q, 0)\right]^{-1} .
\end{aligned}
$$

Using the plasmon-pole susceptibility $\chi^{p p}$ in the phonon self-energy $\Pi^{e p}$ gives a phonon Green's function with poles at the two frequencies $\omega_{+}$and $\omega_{-}$,

$$
\begin{aligned}
D(q, \omega) & =\frac{2 \omega_{T O}\left(\omega^{2}-\tilde{\omega}^{2}\right)}{\hbar\left(\omega^{2}-\omega_{+}^{2}\right)\left(\omega^{2}-\omega_{-}^{2}\right)} \\
\omega_{ \pm}^{2} & =\frac{1}{2}\left\{\omega_{L O}^{2}+\tilde{\omega}_{p}^{2} \pm\left[\left(\omega_{L O}^{2}-\tilde{\omega}_{p}^{2}\right)^{2}+4 \omega_{p}^{2}\left(\omega_{L O}^{2}-\omega_{T O}^{2}\right)\right]^{1 / 2}\right\} .
\end{aligned}
$$

In the plasmon-pole model for the coupled modes, the phonon spectral function $-\pi \operatorname{Im}[D(q, \omega)]$ appearing in the electron-phonon collision term (48) has delta function peaks at $\omega_{+}$and $\omega_{-}$,

$$
\begin{array}{r}
\operatorname{Im}[D(q, \omega)]=\operatorname{Im}\left[D^{+}(q, \omega)+D^{-}(q, \omega)\right] \\
\operatorname{Im}\left[D^{ \pm}(q, \omega)\right]=\mp \frac{\pi \omega_{T O}\left(\omega^{2}-\tilde{\omega}_{p}^{2}\right)}{\hbar \omega_{ \pm}\left(\omega_{+}^{2}-\omega_{-}^{2}\right)}\left[\delta\left(\omega+\omega_{ \pm}\right)-\delta\left(\omega-\omega_{ \pm}\right)\right] .
\end{array}
$$

At low densities, the low-energy hybrid mode has frequency $\omega$ - close to the uncoupled plasmon frequency $\tilde{\omega}_{p}$, but has nonzero weight in the phonon spectral function determined by the factor $\left(\omega_{-}^{2}-\tilde{\omega}_{p}^{2}\right) /\left(\omega_{+}^{2}-\omega_{-}^{2}\right)$. Even though this factor is small at low densities, the low-energy mode has an exponentially larger thermal occupation $N^{0}\left(\omega_{-}\right)$ at low temperatures than the high-energy hybrid mode or uncoupled phonon mode do. Therefore, for low densities and temperatures, the presence of $N^{0}$ in (48) suggests that the mobility calculated by including scattering from the coupled modes should be lower than the mobility calculated by using the uncoupled mode approximation. In addition, "antiscreening" effects due to the dynamic nature of the system response can generate screened potentials that are actually greater than in the unscreened case [27]. Reference [7] presents numerical results for n-type GaAs which show that including dynamic screening, mode coupling, and electron-electron scattering does significantly lower calculated mobilities in situations where ionized impurity scattering does not dominate, as in modulation-doped structures.

\section{CONCLUSION}

The total dielectric function $\epsilon_{T}(q, \omega)$ lends itself to a simple and general method for deriving the inelastic collision term in the electron Boltzmann equation for scattering from a coupled electron-phonon system. When the Born approximation is valid, the inelastic differential scattering rate $W^{\text {inel }}$ can be expressed in terms of the nonequilibrium total dielectric function $\epsilon_{T}$, which includes screening by both electrons and phonons. In the RPA, $W^{\text {inel }}$ separates into a purely electron-electron interaction plus an electron-phonon term describing interactions with the hybrid modes. These interactions are dynamically screened by only the electrons. Reference [7] investigates the effects of the phonon self-energy, dynamical screening, and electron-electron scattering on the mobility in n-type GaAs by means of a numerical solution of the linearized Boltzmann equation.

Because the total dielectric function approach to the Boltzmann equation treats the electrons and ions in a unified manner, it can be applied quite generally to a wide variety of coupled systems. More accurate and complete treatments 8,9] of the lattice part of $\epsilon_{T}^{-1}$ could be useful for more complex electron-phonon systems. Also, it would be very interesting to try to include electron-impurity scattering through the $\epsilon_{T}(q, \omega)$ for an imperfect crystal with local phonon modes. Another logical direction to pursue would start from a dielectric function which goes beyond the RPA to include electron exchange effects in order to derive the exchange scattering term in the Boltzmann equation. However, a systematic approach to improving on the RPA is required, since exchange corrections can be largely canceled by correlation terms. 


\section{ACKNOWLEDGMENTS}

I thank P.B. Allen, D.R. Penn, G.W. Bryant, G.D. Mahan, S. Das Sarma, and J.R. Lowney for helpful conversations.

[1] L.P. Kadanoff and G. Baym, Quantum Statistical Mechanics (Benjamin, Menlo Park, 1962).

[2] T. Holstein, Ann. Phys. 29, 410 (1964).

[3] D.F. DuBois, in Lectures in Theor. Physics, Vol. IX C, ed. W.E. Brittin (Gordon and Breach, New York, 1967$)$ p.469.

[4] V. Špička and P. Lipavský, Phys. Rev. Lett. 73, 3439 (1994).

[5] L. Van Hove, Phys. Rev. 95, 249 (1954).

[6] M.E. Kim, A. Das, and S.D. Senturia, Phys. Rev. B 18, 6890 (1978).

[7] B.A. Sanborn, "Electron-electron interactions, coupled plasmon-phonon modes, and mobility in n-type GaAs," submitted to Phys. Rev. B.

[8] D.R. Penn, S.P. Lewis, and M.L. Cohen, submitted to Phys. Rev. B; E.G. Maksimov, Zh. Eksp. Teor. Fiz. 69, 2236 (1975); [Sov. Phys.-J.E.T.P. 42, 1138 (1976)].

[9] P.B. Allen, M.L. Cohen, and D.R. Penn, Phys. Rev. B. 38, 2513 (1988).

[10] G.D. Mahan Many Particle Physics, (Plenum, New York, 1990), Section 6.3.

[11] P.Lugli and D.K. Ferry, Physica 129B, 532 (1985).

[12] J.R. Lowney and H.S. Bennett, J. Appl. Phys. 69, 7102 (1991); W. Walukiewicz, L. Lagowski, L. Jastrzebski, M. Lichtensteiger, and H.C. Gatos, J. Appl. Phys. 50, 899 (1979); W. Walukiewicz, J. Lagowski, and H.C. Gatos, J. Appl. Phys. 53, 769 (1982).

[13] D. Chattopadhyay, J. Appl. Phys. 53, 3330 (1982).

[14] R. Jalabert and S. Das Sarma, Phys. Rev. B 41, 3651 (1990).

[15] S. Das Sarma, J.K. Jain, and R. Jalabert, Phys. Rev. B 41, 3561 (1990).

[16] X.L. Lei and M.W. Wu, Phys. Rev. B 47, 13338 (1993).

[17] A.W. Overhauser, Phys. Rev. B 3, 1888 (1971); B.I. Lundqvist, Phys. Kondens. Mater. 6, 193 (1967); 6, 206 (1967).

[18] S. Das Sarma, J.K. Jain, and R. Jalabert, Phys. Rev. B 37, 4560 (1988); 37, 6290 (1988).

[19] This method is explained in section 6-2 of reference [1].

[20] B.B. Varga, Phys. Rev. 137, A1896 (1965).

[21] The derivation given in reference [10] for the equilibrium system also holds for the nonequilibrium case.

[22] H.W. Wyld and D. Pines, Phys. Rev. 127, 1851 (1962).

[23] Y.L. Klimontovich, The Kinetic Theory of Electromagnetic Processes (Springer-Verlag, Berlin, 1983); Sov. Phys. JETP 48, 852 (1979).

[24] D. Pines and J.R. Schrieffer, Phys. Rev. 125, 804 (1962).

[25] M.V. Fischetti, Phys. Rev. B 44, 5527 (1991).

[26] J.M. Ziman, Electrons and Phonons (Clarendon Press, Oxford, 1960).

[27] H. Ehrenreich, J. Phys. Chem. Solids 8, 130 (1959). 